\title{
Neuroinflammation in Late-Onset Schizophrenia: Viewing from the Standpoint of the Microglia Hypothesis
}

\author{
Akira Monji Yoshito Mizoguchi \\ Department of Psychiatry, Faculty of Medicine, Saga University, Saga, Japan
}

\section{Keywords}

Late-onset schizophrenia · Microglia · Neuroinflammation · Cytokine $\cdot$ Oxidative stress

\begin{abstract}
Schizophrenia develops mainly in adolescence, but late-onset schizophrenia (LOS) is not uncommon. According to the international consensus, schizophrenia which develops over 40 years old is called LOS and psychosis which develops over 60 years old is called very late-onset schizophrenia-like psychosis (VLOS). Compared to early-onset schizophrenia (EOS) that develops before the age of 40 years, LOS and VLOS are reported to be more common in women, and there are clinically clear differences such as less involvement of genetic factors than EOS. This review outlines the abnormalities of the neuroimmune system in the pathophysiology of LOS, especially focusing on the role of microglia.
\end{abstract}

๑) 2021 S. Karger AG, Basel

\section{Introduction}

Schizophrenia is a chronic and serious mental illness that affects about $1 \%$ of the total population and imposes significant costs on affected patients and their

karger@karger.com

(c) 2021 S. Karger AG, Basel

www.karger.com/nps

Karger! families. The main symptoms are psychotic symptoms such as hallucinations and delusions, decreased motivation/spontaneity, and cognitive decline. Generally, it develops from puberty to adolescence, and the malefemale ratio is approximately $1: 1$. Hypotheses such as hyperdopaminergic function in the limbic system, decreased dopaminergic function in the frontal cortex, or decreased excitatory amino acid function have been pointed out, but the etiology of schizophrenia remains unclear. There are many. Although it has been pointed out that genetic factors are involved in schizophrenia, no gene has a strong effect on increasing the risk of developing schizophrenia. It is presumed that schizophrenia develops due to environmental factors in the early life, including the embryonic period, and childhood and adolescents based on a predisposition to schizophrenia. Recent studies have often pointed out that neuroinflammation, or the role of the neuroimmune system, plays an important in the pathophysiology of schizophrenia. We have conducted studies focusing on microglia, which play a central role in innate immunity in the central nervous system (CNS) and have reported on schizophrenia and neuroinflammation $[1,2]$. This article outlines the relationship between neuroinflammation in the pathophysiology of late-onset schizophrenia (LOS). 
Table 1. Clinical differences in schizophrenia at each age onset

\begin{tabular}{llll}
\hline Features & EOS & LOS & VLOS \\
\hline Age of onset, years & $<40$ & $40-60$ & $>60$ \\
Female preponderance & No & Yes & Definitely \\
Negative symptoms & Definitely & May be & Less likely \\
Learning & Ok & Ok & Impaired \\
Retention & Ok & Ok & Impaired \\
Progressive cognitive deterioration & Yes & Yes & Yes (very high) \\
Brain structural abnormalities & No & No & Yes \\
Family history & Present & Present & Weak association \\
Early childhood maladjustments & Present & Present & Absent \\
Antipsychotic dosing & Higher & Lower & Lower \\
Risk of tardive dyskinesia & Present & Present & Very high \\
\hline
\end{tabular}

Author's modification from Tampi et al. [4]. EOS, early-onset ( $<40$ years of age) schizophrenia; LOS, late-onset ( $>40$ years of age) schizophrenia; VLOS, very late-onset ( $>60$ years of age) schizophrenia-like psychosis.

\section{Characteristics of Senile-Onset Schizophrenia}

Psychosis that develops in old age is increasing its clinical importance with the aging of the population. Approximately $60 \%$ of senile-onset psychosis is secondary psychosis caused by neurodegenerative diseases, including dementia, prescription drugs, and delirium. Therefore, clinicians must distinguish secondary psychosis from senile-onset schizophrenia. Adolescent onset is common in schizophrenia, but senile-onset schizophrenia is by no means rare. The pathophysiology of LOS varies, and it develops through complex interactions such as biological, psychological, social, and environmental factors. According to the international consensus, schizophrenia which develops over 40 years is called LOS and psychosis which develops over 60 years old is called very late-onset schizophrenia-like psychosis (VLOS). Compared to early-onset schizophrenia (EOS), which develops before the age of 40, LOS and VSOS LP are said to be more common in women and genetic factors are less involved than EOS. In addition, while delusions of reference that occur in relationships with family members and neighbors tend to occupy the center of the disease image, treatment responsiveness and outcomes are generally considered to be good. As shown in Table 1, there are clinically distinct differences among EOS and LOS and VLOS, especially between EOS and VLOS [3-7]. Treatment of LOS requires individual treatment due to its characteristics, especially drug selection in consideration of complications such as lifestylerelated diseases, and appropriate combination of nondrug therapy is also required $[4,8]$. In addition, although the necessity of distinguishing senile-onset schizophrenia, or VLOS from neurodegenerative diseases was mentioned above, it is necessary to keep in mind that schizophrenia itself is a risk factor for developing dementia for long-term treatment [9].

\section{Outlines of the Microglial Hypothesis of Schizophrenia}

Microglia, which account for about $10 \%$ of the total number of cells in the CNS, are derived from the mesoderm like blood and immune system cells and were thought to be monocyte-macrophage lineage cells. However, it has recently been reported that microglia originate from precursor cells generated in embryonic oval sac and they have a gene expression pattern different from those of bone marrow-derived monocytes and macrophages. As "brain macrophages," microglia play a central role in the innate immunity of the CNS, and activated microglia are a major source of cytokines and free radicals. There is also the idea of conveniently dividing the direction of activation into tissue-damaging M1 and tissue-protecting M2. Inactivated microglia are called "resting microglia," and their role has been unknown for a long time, but recent studies have revealed that they also play an important role in the development of the CNS and maintenance of homeostasis and are now called "surveying microglia" $[10,11]$. The conceptual scheme of microglia in the pathophysiology of schizophrenia is as shown in Figure 1. At present, the pathophysiology of schizophrenia and the neuroinflammation or neuroimmune system centered on microglia. Regarding the abnormalities of neuroimmnune 


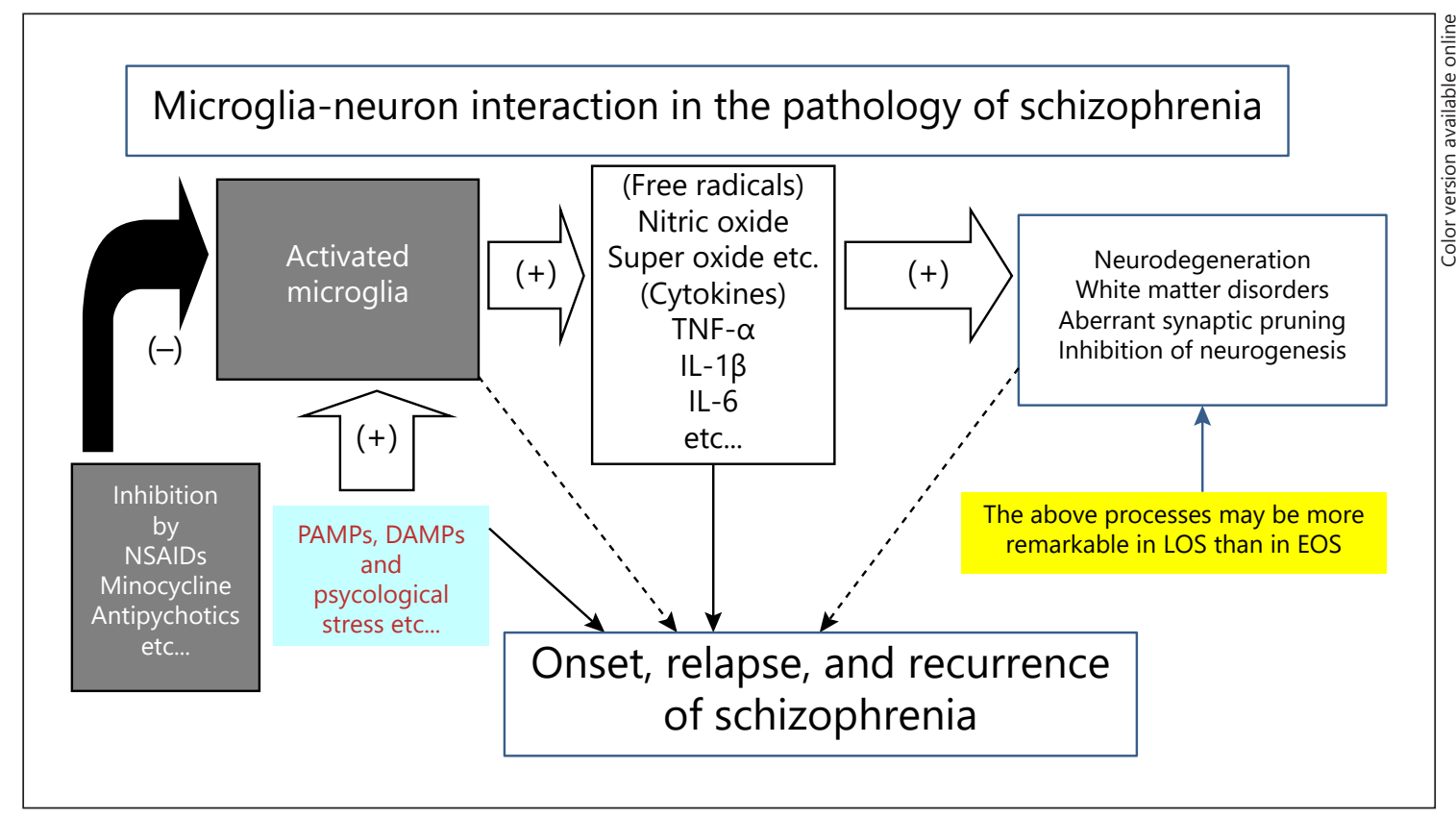

Fig. 1. Conceptual scheme of the role of microglia in the pathophysiology of schizophrenia. Author's modification from Monji et al. [2]. PAMPs, pathogen-associated molecular patterns; DAMPs, danger-associated molecular patterns; (+), promotion; (-), inhibition; EOS, early-onset schizophrenia; LOS, late-onset schizophrenia.

systems in schizophrenia, the following points are mainly mentioned [10-14]:

1. In schizophrenia, the expression of inflammatory cytokines and related molecules is increased in the initial stage and the acute stage, and decreased after the drug treatment.

2. In a PET study of schizophrenia using a ligand that detects TSPO (translocator protein) that is strongly expressed in activated microglia, microglial activation was significantly enhanced compared to the healthy subject group and these findings are also found in the high-risk group for psychosis.

3. The higher the peripheral blood concentration of CRP, a typical inflammatory marker, the more remarkable the cognitive impairment of schizophrenia.

4. In the postmortem brain of schizophrenia, the density of microglia is significantly higher in the frontal cortex and hippocampus than in the healthy subject group.

5. Infection during pregnancy, malnutrition, and trauma during childbirth increase the risk of developing schizophrenia.

6. Microglia play a major role in "synaptic pruning," which is important for the pathology of schizophrenia

7. Anti-inflammatory therapy targeting neuroinflammation (with antipyretic analgesics and anti-cytokine an- tibodies), antioxidant therapy, and minocycline, which has an inhibitory effect on microglial activation, are effective for schizophrenia.

8. Some antipsychotics have a neuroinflammation-regulating effect, especially an inhibitory effect of microglial activation.

According to a report focusing on the relationship between schizophrenia onset in old age and neuroinflammation, high peripheral blood CRP levels increase the risk of developing LOS and VLOS by 6-10 times [15]. In the next section, we will discuss some points that have received particular attention in recent years regarding schizophrenia and neuroinflammation in relation to LOS.

\section{A PET Study of Schizophrenia on Microglial Activation}

The aforementioned TSPO has a molecular weight of $18 \mathrm{kDa}$ and was once called a peripheral benzodiazepine receptor. TSPO is abundant in peripheral organs such as the adrenal gland and kidney, and its presence in the CNS is relatively poor. By utilizing the phenomenon that TSPO expression is remarkable in the mitochondria of activated 
microglia, PET imaging using a TSPO ligand is performed to detect activated microglia. In a study using $\left[{ }^{11} \mathrm{C}\right]$ PK11195 as a ligand, van Berckel et al. [16] reported that microglial activation was significantly stronger in the entire gray matter of schizophrenic patients within 5 years after onset of schizophrenia than in healthy subjects. Doorduin et al. [17] reported that the same ligand was used to significantly enhance microglial activation in the hippocampus of schizophrenic patients. In a study using another TSPO ligand, $\left[{ }^{11} \mathrm{C}\right]$ DAA1106, Takano et al. [18] reported that the degree of microglial activation throughout the cortex in patients with chronic schizophrenia was not significantly different from that in healthy subjects, while the degree of increased microglial activation has a significant positive correlation with positive symptom scores and duration of illness. In a study using the secondgeneration TSPO ligand $\left[{ }^{18} \mathrm{~F}\right]$ FEPPA, Kenk et al. [19] reported that no significant increase in microglial activation was observed in comparison to healthy subjects, while these results were due to the effect of antipsychotic drugs on suppressing microglial activation. In a study by Bloomfield et al. [20] using another second-generation TSPO ligand, $\left[{ }^{11} \mathrm{C}\right]$ PBR28, microglial activation was significantly higher than in healthy individuals, not only in schizophrenia patients, but even in the high-risk group of schizophrenia. In addition, a study using the same ligand by Selvaraj et al. [21] has shown a correlation between increased microglial activation and decreased cortical volume. The above reports support a significant increase in microglial activation in patients with schizophrenia, but recent reports have denied these results $[22,23]$. The heterogeneity conundrum of TSPO PET Imaging in psychiatric disorders including schizophrenia and major depressive disorders has been discussed in recent relevant reviews $[24,25]$. To our knowledge, there is no microglial PET study focusing only on LOS, but a recent study focusing on changes in microglial activation over time in schizophrenia has shown TSPO binding ability to change with age [26]. Therefore, it is expected that research will progress in cross-sectional and longitudinal comparison of microglial activation changes among EOS, LOS, and VLOS [27].

\section{Microglial Synaptic Pruning and Schizophrenia}

Conventionally, inactivated microglia are called "resting microglia," and their role has been unknown, but recent studies have revealed that they also play an important role in the development of the CNS and the mainte-

Late-Onset Schizophrenia and

Neuroinflammation nance of homeostasis. By using the imaging method, using a two-photon excitation microscope, it has been reported that the dynamics of the microglial processes are very dynamic, and that they are constantly retracted within a certain region and that they repeatedly contact with synapses in the live mouse cerebral cortex. In this phenomenon, microglia perform an appropriate management function of synapses called synaptic pruning, specifically, the action of removing synapses that are no longer needed during the developmental stage of the CNS [10, 28-30]. Therefore, the abnormal conditions of synaptic pruning carried by microglia and its correction are considered to be targets for the treatment of a wide range of neuropsychiatric disorders [10, 30]. Sekar et al. [31] found that microglial synaptic pruning is regulated by complement $\mathrm{C} 4$, and that the genetic polymorphism is involved in the risk of developing schizophrenia. The stronger the complement $\mathrm{C} 4$ activity is, the higher the risk of developing schizophrenia is. In addition, excessive synaptic pruning in schizophrenia has been demonstrated by a tissue model derived from the schizophrenic brain [32]. Excessive synaptic pruning during the onset of schizophrenia is considered to be an important target for suppressing the onset of the disease and delaying the progression of the disease. Interestingly, this excessive synaptic pruning phenomenon has also been found in Alzheimer's disease [33]. Traditionally, there have been discussions on the pathophysiology of schizophrenia from the perspective of "accelerated aging" [34-36] and a recent report suggested the involvement of microglia in these processes [26]. Excessive synaptic pruning phenomenon will thus be important targets for the diagnosis and treatment of LOS in the future.

\section{Possibility of Schizophrenia Treatment Targeting Neuroinflammation}

If neuroinflammation contributes to the pathophysiology of schizophrenia, the effect of anti-inflammatory therapy is naturally expected. Non-steroidal anti-inflammatory drugs, antioxidants, anti-cytokine therapy targeting the inflammatory cytokines TNF- $\alpha$, and IL-6, or minocycline, which has an inhibitory effect on microglial activation, are added as an adjunctive therapy and it has a certain effect. Regarding the subtypes or stages for which anti-inflammatory therapy is effective, treatmentresistant cases, severe cases, and cases showing suiciderelated behavior are assumed. Accumulation of further knowledge including the biomarkers for detecting neuro- 
inflammation is essential for clinical application of antiinflammatory therapy [37]. Oxidative stress by redox dysregulation, a disturbance of balance between oxidants and antioxidant species, is known to play an important role in the pathophysiology of schizophrenia and it is closely related to neuroinflammation through microglial activation $[38,39]$. Whether or not the effect of anti-inflammatory or anti-oxidant therapy is different among EOS, LOS, and VLOS should also be clarified.

It is a well-known fact that chronic inflammation is the basis of the pathophysiology of metabolic syndrome including diabetes [40]. On the other hand, schizophrenia has a predisposition to be complicated with metabolic syndrome such as diabetes. A recent report has also shown inflammation to be a common cause of insulin resistance and schizophrenia [41]. The formation of advanced glycation end products (AGEs) is a key pathophysiological event linked to the onset and progression of diabetic complications. Oxidative stress leverages the formation of AGEs, which is also called "carbonyl stress." It has been reported that $20 \%$ of schizophrenia patients reportedly exhibit increased blood levels of pentosidine, one of AGEs and that lowered levels of vitamin B6 and that the administration of vitamin B6 pyridoxamine to patients with schizophrenia under enhanced carbonyl stress can effectively ameliorate their symptoms $[42,43]$. Despite advances in psychiatry, the lifespan difference between psychiatric patients including schizophrenia and healthy individuals is widening, and the main cause of the lifespan difference is cardiovascular disorders caused by metabolic syndrome such as diabetes [44]. It is also well known that most psychotropic drugs, including antipsychotics, have weight gain effects and that cognitive impairment is more pronounced in schizophrenia with diabetes [45]. The above points are more clinically problematic in LOS.

\section{Conclusion}

As mentioned above, LOS, especially VLOS, has a clear clinical difference from EOS. We will finish the article with the hope that the pathophysiology of LOS will be elucidated from the perspective of "neuroinflammation" and that it will become a breakthrough in the diagnosis and treatment of LOS.

\section{Conflict of Interest Statement}

The authors have no conflicts of interest to declare.

\section{Funding Sources}

There are no funding sources related to the manuscript.

\section{Author Contributions}

Akira Monji created the concept and wrote the manuscript. Yoshito Mizoguchi reviewed and critiqued the manuscript. Both authors read and approved the final version of the manuscript.

\section{References}

1 Monji A, Kato T, Kanba S. Cytokines and schizophrenia: microglia hypothesis of schizophrenia. Psychiatry Clin Neurosci. 2009;63(3):257-65.

2 Monji A, Kato TA, Mizoguchi Y, Horikawa H, Seki Y, Kasai M, et al. Neuroinflammation in schizophrenia especially focused on the role of microglia. Prog Neuropsychopharmacol Biol Psychiatry. 2013;42:115-21.

3 Assche LV, Morrens M, Luyten P, de Ven LV, Vandenbulcke M. The neuropsychology and neurobiology of late-onset schizophrenia and very-late-onset schizophrenia-like psychosis: a critical review. Neurosci Biobehav Rev. 2017;83:604-21.

4 Tampi RR, Young J, Hoq R, Resnick K, Tampi DJ. Psychotic disorders in late life: a narrative review. Ther Adv Psychopharmacol. 2019;9:1-13.
5 Howard R, Rabins PV, Seeman MV, Jeste DV. The International Late-Onset Schizophrenia Group, 2000. Late-onset schizophrenia and very-late-onset schizophrenia-like psychosis: an international consensus. Am J Psychiatry. 2000;157:172-8.

6 Cohen CI, Meesters PD, Zhao J. New perspectives on schizophrenia in later life: implications for treatment, policy, and research. Lancet Psychiatry. 2015;2(4):340-50.

7 Reinhardt MM, Cohen CI. Late-life psychosis: diagnosis and treatment. Curr Psychiatry Rep. 2015;17(2):1.

8 Howard R, Cort E, Bradley R, Harper E, Kelly L, Bentham P, et al. Antipsychotic treatment of very late-onset schizophrenia-like psychosis (ATLAS): a randomised, controlled, double-blind trial. Lancet Psychiatry. 2018;5(7): 553-63.
9 Cai L, Huang J. Schizophrenia and risk of dementia: a meta-analysis study. Neuropsychiatr Dis Treat. 2018;14:2047-55.

10 Wake $\mathrm{H}$, Moorhouse AJ, Miyamoto A, Nabekura J. Microglia: actively surveying and shaping neuronal circuit structure and function. Trends Neurosci. 2013;36(4):209-17.

11 Tian L, Hui CW, Bisht K, Tan Y, Sharma K, Chen S, et al. Microglia under psychosocial stressors along the aging trajectory: consequences on neuronal circuits, behavior, and brain diseases. Prog Neuropsychopharmacol Biol Psychiatry. 2017;79(Pt A):27-39.

12 Mondelli V, Vernon AC, Turkheimer F, Dazzan P, Pariante CM. Brain microglia in psychiatric disorders. Lancet Psychiatry. 2017; 4(7):563-72.

13 Khandaker GM, Dantzer R, Jones PB. Immunopsychiatry: important facts. Psychol Med. 2017;47(13):2229-37. 
14 Pape K, Tamouza R, Leboyer M, Zipp F. Immunoneuropsychiatry - novel perspectives on brain disorders. Nat Rev Neurol. 2019; 15(6):317-28.

15 Wium-Andersen MK, Orsted DD, Nordestgaard BG. Elevated C-reactive protein associated with late- and very-late-onset schizophrenia in the general population: a prospective study. Schizophrenia Bull. 2014;40: 1117-27.

16 van Berckel BN, Bossong MG, Boellaard R, Kloet R, Schuitemaker A, Caspers E, et al. Microglia activation in recent-onset schizophrenia: a quantitative (R)-[11C]PK11195 positron emission tomography study. Biol Psychiatry. 2008;64(9):820-2.

17 Doorduin J, de Vries EF, Willemsen AT, de Groot JC, Dierckx RA, Klein HC. Neuroinflammation in schizophrenia-related psychosis: a PET study. J Nucl Med. 2009;50(11) 1801-7.

18 Takano A, Arakawa R, Ito H, Tateno A, Takahashi $\mathrm{H}$, Matsumoto R, et al. Peripheral benzodiazepine receptors in patients with chronic schizophrenia: a PET study with [11C] DAA1106. Int J Neuropsychopharmacol. 2010;13(7):943-50

19 Kenk M, Selvanathan T, Rao N, Suridjan I, Rusjan P, Remington G, et al. Imaging neuroinflammation in gray and white matter in schizophrenia: an In-Vivo PET Study with [18F]-FEPPA. Schizophr Bull. 2015;41:8593.

20 Bloomfield PS, Selvaraj S, Veronese M, Rizzo G, Bertoldo A, Owen DR, et al. Microglial activity in people at ultra high risk of psychosis and in schizophrenia: an [(11)C]PBR28 PET brain imaging study. Am J Psychiatry. 2016; 173(1):44-52.

21 Selvaraj S, Bloomfield PS, Cao B, Veronese M Turkheimer F, Howes OD. Brain TSPO imaging and gray matter volume in schizophrenia patients and in people at ultra high risk of psychosis: an [11C]PBR28 study. Schizophr Res. 2018;195:206-14.

22 Plavén-Sigray P, Matheson GJ, Collste K, Ashok AH, Coughlin JM, Howes OD, et al. Positron emission tomography studies of the glial cell marker translocator protein in patients with psychosis: a meta-analysis using individual participant data. Biol Psychiatry. 2018;84(6):433-42.
23 Sneeboer MAM, van der Doef T, Litjens M, Psy NBB, Melief J, Hol EM, et al. Microglial activation in schizophrenia: is translocator $18 \mathrm{kDa}$ protein (TSPO) the right marker? Schizophr Res. 2020;215:167-72.

24 De Picker L, Morrens M. Perspective: solving the heterogeneity conundrum of TSPO PET imaging in psychosis. Front Psychiatry. 2020; 11:362.

25 Meyer JH, Cervenka S, Kim MJ, Kreisl WC, Henter ID, Innis RB. Neuroinflammation in psychiatric disorders: PET imaging and promising new targets. Lancet Psychiatry. 2020;7(12):1064-74.

26 De Picker L, Ottoy J, Verhaeghe J, Deleye S, Wyffels L, Fransen E, et al. State-associated changes in longitudinal [18F]-PBR111 TSPO PET imaging of psychosis patients: evidence for the accelerated ageing hypothesis? Brain Behav Immun. 2019;77:46-54.

27 Plavén-Sigray P, Matheson GJ, Coughlin JM, Hafizi S, Laurikainen H, Ottoy J, et al. Metaanalysis of the glial marker TSPO in psychosis revisited: reconciling inconclusive findings of patient-control differences. Biol Psychiatry. 2021;89(3):e5-8.

28 Vilalta A, Brown GC. Neurophagy, the phagocytosis of live neurons and synapses by glia, contributes to brain development and disease. FEBS J. 2018;285(19):3566-75.

29 Tian L, Hui CW, Bisht K, Tan Y, Sharma K, Chen S, et al. Microglia under psychosocial stressors along the aging trajectory: consequences on neuronal circuits, behavior, and brain diseases. Prog Neuropsychopharmacol Biol Psychiatry. 2017;79(Pt A):27-39.

30 Paolicelli RC, Bolasco G, Pagani F, Maggi L, Scianni M, Panzanelli P, et al. Synaptic pruning by microglia is necessary for normal brain development. Science. 2011;333(6048):14568.

31 Sekar A, Bialas AR, de Rivera H, Davis A, Hammond TR, Kamitaki N, et al. Schizophrenia risk from complex variation of complement component 4. Nature. 2016;530(7589): $177-83$.

32 Sellgren CM, Gracias J, Watmuff B, Biag JD, Thanos JM, Whittredge PB, et al. Increased synapse elimination by microglia in schizophrenia patient-derived models of synaptic pruning. Nat Neurosci. 2019;22(3):374-85.

33 Rajendran L, Paolicelli RC. Microglia-mediated synapse loss in Alzheimer's Disease. J Neurosci. 2018;38(12):2911-9.

34 Kirkpatrick B, Kennedy BK. Accelerated aging in schizophrenia and related disorders: future research. Schizophr Res. 2018;196:4-8.
35 Douaud G, Groves AR, Tamnes CK, Westlye LT, Duff EP, Engvig A, et al. A common brain network links development, aging, and vulnerability to disease. Proc Natl Acad Sci U S A. 2014;111(49):17648-53.

36 Nour MM, Howes OD. Interpreting the neurodevelopmental hypothesis of schizophrenia in the context of normal brain development and ageing. Proc Natl Acad Sci U S A. 2015 112(21):E2745

37 Müller N. COX-2 inhibitors, aspirin, and other potential anti-inflammatory treatments for psychiatric disorders. Front Psychiatry. 2019; 10:375.

38 Perkins DO, Jeffries CD, Do KQ. Potential roles of redox dysregulation in the development of schizophrenia. Biol Psychiatry. 2020; 88(4):326-36.

39 Kato TA, Monji A, Yasukawa K, Mizoguchi Y, Horikawa H, Seki Y, et al. Aripiprazole inhibits superoxide generation from phorbol-myristate-acetate (PMA)-stimulated microglia in vitro: implication for antioxidative psychotropic actions via microglia. Schizophr Res. 2011;129(2-3):172-82.

40 Hotamisligil GS. Inflammation and metabolic disorders. Nature. 2006;444(7121):860-7.

41 Perry BI, Burgess S, Jones HJ, Zammit S, Upthegrove R, Mason AM, et al. The potential shared role of inflammation in insulin resistance and schizophrenia: a bidirectional twosample mendelian randomization study. PLoS Med. 2021;18(3):e1003455.

42 Ohnuma T, Nishimon S, Takeda M, Sannohe T, Katsuta N, Arai H. Carbonyl stress and microinflammation-related molecules as potential biomarkers in schizophrenia. Front Psychiatry. 2018;9:82

43 Toyoshima M, Jiang X, Ogawa T, Ohnishi T, Yoshihara S, Balan S, et al. Enhanced carbonyl stress induces irreversible multimerization of CRMP2 in schizophrenia pathogenesis. Life Sci Alliance. 2019;2(5):e201900478.

44 Lawrence D, Hancock KJ, Kisely S. The gap in life expectancy from preventable physical illness in psychiatric patients in Western Australia: retrospective analysis of population based registers. BMJ. 2013;346:f2539.

45 MacKenzie NE, Kowalchuk C, Agarwal SM, Costa-Dookhan KA, Caravaggio F, Gerretsen $\mathrm{P}$, et al. Antipsychotics, metabolic adverse effects, and cognitive function in schizophrenia. Front Psychiatry. 2018;9:62.
Late-Onset Schizophrenia and Neuroinflammation
Neuropsychobiology 2022;81:98-103

DOI: $10.1159 / 000517861$ 